\title{
Enfrentando o estresse em tempos de pandemia: proposição de uma Cartilha
}

\section{Coping with stress in times of pandemic: a booklet proposal}

\author{
Sônia Regina Fiorim ENUMO ${ }^{1}$ iD) 0000-0001-9038-6151

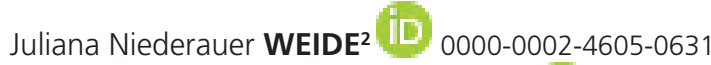 \\ Eliana Cristina Chiminazzo VICENTINI ${ }^{1}$ iD) 0000-0001-9463-0801 \\ Murilo Fernandes de ARAUJO ${ }^{1}$ iD 0000-0003-1707-4973 \\ Wagner de Lara MACHADO2 ${ }^{2}$ (D) 0000-0001-5555-5116
}

\section{Resumo}

A pandemia de COVID-19 está sendo um grande estressor. A autorregulação emocional e comportamental é alterada quando sob ameaça/desafio frente às três necessidades psicológicas básicas: competência, relacionamento e autonomia. O conhecimento psicológico, especialmente da Teoria Motivacional do Coping sobre processos de enfrentamento do estresse e suas consequências na saúde física e mental, embasou a proposição de uma Cartilha que visa à promoção da saúde e bem-estar. O material foi avaliado online por oito juízes quanto à compreensão da linguagem, conteúdo e pertinência. A versão final ficou com dezesseis páginas, ilustradas, estando organizada em três seções: Introdução, Identificação de respostas de estresse e Estratégias de enfrentamento referentes às necessidades psicológicas básicas. Para cada uma delas, a Cartilha apresenta três quadros, apontando "Dificuldades", "O que evitar" e "O que pode ser feito", além de um espaço para anotações. Esta tradução de conhecimento científico em material de divulgação e psicoeducação pode ser útil para leigos e para uso em serviços de saúde, estando disponível em mídias sociais.

Palavras-chave: Autorregulação; Coping; Estresse; Pandemias; Psicologia da saúde.

\footnotetext{
$\boldsymbol{\nabla} \boldsymbol{\nabla} \boldsymbol{\nabla}$
}

1 Pontifícia Universidade Católica de Campinas (PUC-Campinas), Centro de Ciências da Vida, Programa de Pós-Graduação em Psicologia. Av. John Boyd Dunlop, s/n., Prédio Administrativo CCV, Jd. Ipaussurama, 13060-904, Campinas, SP, Brasil. Correspondência para/Correspondence to: S.R.F. ENUMO.E-mail: <sonia.enumo@puc-campinas.edu.br>.

2 Pontifícia Universidade Católica do Rio Grande do Sul, Escola de Ciências da Saúde e da Vida, Programa de Pós-Graduação em Psicologia. Porto Alegre, RS, Brasil

$\boldsymbol{\nabla} \boldsymbol{\nabla} \boldsymbol{V}$

Como citar este artigo/How to cite this article

Enumo, S. R. F., Weide, J. N., Vicentini, E. C. C., Araujo, M. F., \& Machado, W. L. (2020). Enfrentando o estresse em tempos de pandemia: proposição de uma Cartilha. Estudos de Psicologia (Campinas), 37, e200065. http://dx.doi.org/10.1590/1982-0 $275202037 \mathrm{e} 200065$ 


\begin{abstract}
The COVID-19 pandemic has been a major stressor. Emotional and behavioral self-regulation can change when there is a threat/challenge to the three basic psychological needs for competence, relatedness and autonomy. Psychological knowledge, especially the Motivational Theory of Coping, about processes of coping with stress and its consequences on physical and mental health supported the proposal of a booklet, aiming to assist in the health and well-being promotion. The material was assessed online by eight judges regarding their understanding of language, content and relevance. The final version has 16 pages, illustrated, organized in three sections: Introduction, Identifying stress responses and Coping strategies related to the basic psychological needs. For each of them, the Booklet presents three charts, pointing out "Difficulties", "What to avoid", "What can be done", and space for notes. This translation of scientific knowledge into promotional and psychoeducational material can be useful for lay people and can be used in health services, and it is available on social media.
\end{abstract}

Keywords: Self-regulation; Coping; Stress; Pandemics; Health psychology.

A recente pandemia de Coronavirus Disease 2019 (COVID-19), assim denominada por ter sido notificada em 2019 pelo governo chinês, foi gerada pelo coronavírus 2, denominado como Severe Acute Respiratory Syndrome Coronavirus 2 (SARS-COV-2) (Liang, 2020). Essa situação transformou-se em um grande desafio para a sociedade. Trata-se de um evento potencialmente estressante, considerando as medidas de prevenção e contenção da doença, bem como seus impactos econômicos, políticos e sociais (Afifi, Felix, \& Afifi, 2012; Correia, Luck, \& Verner, 2020; van Bavel et al., 2020). Não menos relevante é o impacto na saúde mental, tendo em vista as alterações emocionais, cognitivas e comportamentais características desse período (Barros-Delben et al., 2020; Benight \& Harper, 2002; Brooks et al., 2020; Center for Disease Control Prevention [CDCP], 2020; Inter-Agency Standing Commitee, 2020; Lunn et al., 2020; Qiu et al., 2020; Shonkoff, 2020).

Considerando a urgência dessa situação e a necessidade de amenizar os impactos na saúde mental, em específico quanto ao acesso da população em geral a conteúdos científicos (World Health Organization [WHO], 2020), foi elaborada a "Cartilha para enfrentamento do estresse em tempos de pandemia" (Weide, Vicentini, Araujo, Machado, \& Enumo, 2020). A Cartilha tem o propósito de contribuir na promoção de estratégias de enfrentamento benéficas e flexíveis, que podem auxiliar na manutenção do bem-estar emocional no dia a dia. A promoção da saúde mental tem sido enfatizada como estratégia complementar às de prevenção e tratamento na Psicologia, em especial após o surgimento da Psicologia Positiva (Keyes, 2007). Essa abordagem é focada no desenvolvimento das potencialidades humanas (Seligman \& Csikszentmihalyi, 2000) e alinha-se à compreensão de que a saúde mental é não apenas a ausência dos transtornos mentais, mas inclui características positivas, como o bem-estar e as estratégias adaptativas de manejo do estresse (e.g., coping) (WHO, 2004). A Cartilha foi elaborada a partir do conhecimento produzido recentemente na Psicologia e áreas afins, buscando traduzir e popularizar o conhecimento científico em uma linguagem mais acessível para facilitar a compreensão da informação por diversos públicos (Aldwin, 2009; Brooks et al., 2020; Correia et al., 2020; Florko, 2020; Hanssen et al., 2015; Liang, 2020; Lunn et al., 2020; Organização Panamericana de Saúde [OPAS], 2015; Shonkoff, 2020; Skinner, Edge, Altman, \& Sherwood, 2003; Skinner \& Zimmer-Gembeck, 2016; van Bavel et al., 2020; Zimmer-Gembeck \& Skinner, 2016; Zimmer-Gembeck et al., 2018).

A Psicologia acumulou um conjunto sólido de conhecimentos sobre o enfrentamento adaptativo do estresse e a regulação das emoções ao longo do desenvolvimento humano (Aldwin, 2009; Folkman, 2011; Lazarus \& Folkman, 1984; Skinner \& Zimmer-Gembeck, 2016). A literatura define como situações estressoras aquelas com as quais o indivíduo se vê com dificuldades para lidar, por estarem além de sua capacidade de enfrentamento, ou aquelas nas quais ele se avalia como impossibilitado de lidar com os conflitos internos

2 gerados por esse evento (Lazarus \& Folkman, 1984). 
Os indicadores mais comuns de estresse e ansiedade são de ordem: (a) física, como dor de cabeça, aumento dos batimentos cardíacos, problemas de alimentação e de sono, úlceras, exaustão física; (b) emocionais, como tristeza, nervosismo, raiva, culpa, preocupação excessiva, perda de vontade e humor deprimido; (c) comportamentais, como irritabilidade, distanciamento, abuso de substâncias, violência; (d) cognitivos, como a perda de memória, dificuldade de concentração, dificuldade de tomar decisões (Aldwin, 2009; Compas, Connor-Smith, Saltzman, Thomsen, \& Wadsworth, 2001; Lazarus \& Folkman, 1984).

No contexto da epidemia da COVID-19, alguns dos principais estressores estão relacionados à duração da quarentena, ao distanciamento social, à frustração e ao tédio, bem como ao acúmulo de tarefas, incluindo a realização de atividades normalmente feitas fora de casa (homeschooling e homeworking, por exemplo), à falta de suprimentos, à inadequação das informações e às dificuldades econômicas. Relacionam-se também à COVID-19, o medo de contrair a doença, a preocupação com a saúde própria e dos entes queridos, o estigma da doença e os riscos do trabalho, no caso de profissionais da saúde e de serviços vitais (Brooks et al., 2020; Qiu et al., 2020; van Bavel et al., 2020).

As reações aos estressores têm relação com sua severidade, duração e outras características, como tipo, permanência (agudo ou crônico), previsibilidade, rapidez de aparecimento (abrupto ou lento), flutuação e intensidade (fraco, moderado, forte, ambíguo). Dependem também de características do sujeito, como a idade, o gênero, as experiências prévias e o temperamento, dentre outras. Relacionam-se ainda às características do contexto, destacando-se a importância da rede de suporte social (Aldwin, 2009).

Apesar de um evento ser avaliado como um estressor a partir da interpretação e do julgamento dos recursos de coping disponíveis pelo indivíduo, o contexto de pandemia tem se mostrado um estressor comum, uma vez que promove incertezas, ameaças à vida e desestabilização de rotinas. O indivíduo está sob estresse quando percebe as situações e os eventos como ameaçadores ou desafiadores de três Necessidades Psicológicas Básicas (NPB), que são inatas, universais e de valor evolutivo adaptativo aos seres humanos: (i) a NPB de "relacionamento" ou pertença, de se sentir aceito e compreendido pelos outros, de ter relações próximas que sejam estáveis, seguras e duradouras; (ii) a NPB de "competência", de se sentir com controle da situação e eficaz para gerenciar desafios e cumprimento de metas e objetivos; e (iii) a NPB de "autonomia", de se manifestar e endossar suas ações ou crenças, de ser capaz de iniciar tarefas ou tomar decisões e assumir as consequências do próprio comportamento (Ryan \& Deci, 2017; Skinner \& Wellborn, 1994). Com a percepção de ameaça ou desafio a essas três NPB, as pessoas ficam vulneráveis ao estresse, pois sua capacidade de autorregulação é desafiada (Ramos, Enumo, \& Paula, 2015; Skinner \& Wellborn, 1994).

Frente a isso, são ativadas uma série de respostas psicológicas e fisiológicas para lidar de alguma forma com essa situação estressora (Folkman, 2011; Lazarus \& Folkman, 1984; Skinner \& Zimmer-Gembeck, 2016; Slavich \& Cole, 2013). As reações a essas ameaças ou desafios podem ser voluntárias ou involuntárias (Compas et al., 2001; Skinner et al., 2003; Skinner \& Wellborn, 1994), levando a um quadro de estresse, que pode ser positivo, tolerável ou tóxico, especialmente frente à falta de suporte social (Shonkoff et al., 2012). A maior duração e intensidade dessas situações estressantes agravam as condições de saúde física e mental, por processos psiconeuroimunológicos e epigenéticos, que alteram a expressão dos genes (Aldwin, 2009; Miller, Chen, \& Parker, 2011; Slavich \& Cole, 2013), dependendo principalmente de como se reage a elas, promovendo a resiliência ou a vulnerabilidade ao estresse ao longo do ciclo vital (Aldwin, 2009; Enumo, Linhares, Machado, \& Silva, 2017; Folkman, 2011; Lazarus \& Folkman, 1984; Skinner \& Zimmer-Gembeck, 2016; Zimmer-Gembeck \& Skinner, 2016).

Nessas condições, são acionados a reatividade biológica do sujeito, as suas características de temperamento e o repertório de estratégias de enfrentamento/coping dos eventos estressores aprendidas 
ao longo da vida nos vários ambientes físicos e sociais. É um processo de autorregulação sob estresse, que inclui a regulação do comportamento, emoções, cognições, reações fisiológicas e motivações (Compas et al., 2001; Skinner et al., 2003; Skinner \& Wellborn, 1994). Contudo, o indivíduo não faz isso sozinho. Depende desde cedo do corregulador, do outro, especialmente do suporte social, emocional e instrumental dos demais (Shonkoff, 2020; Skinner \& Zimmer-Gembeck, 2016; Zimmer-Gembeck \& Skinner, 2011); por isso é tão importante cuidar das relações interpessoais e ter um relacionamento de mútuo apoio, seja emocional ou prático, especialmente em situações de crise (Shonkoff, 2020).

A Cartilha apresentada neste trabalho ilustra a possibilidade de elaboração de materiais práticos/ aplicados com base em estudos científicos no contexto da Psicologia aplicada à área da saúde. Esse material pode subsidiar uma prática baseada em evidências, garantindo a qualidade dos serviços psicológicos prestados à população. Essa é uma forma de "[...] auxiliar no processo de construção do conhecimento e da difusão de informações científicas que possam orientar o cuidado oferecido aos indivíduos de forma efetiva e ética" (Melnik, Souza, \& Carvalho, 2014, p.79).

\section{Método}

\section{Desenvolvimento do instrumento e referencial teórico}

Adotou-se a perspectiva mais recente sobre processo de estresse e seu enfrentamento (coping, em inglês), que entende o coping como a autorregulação sob estresse (Compas et al., 2001; Skinner et al., 2003; Skinner \& Wellborn, 1994; Skinner \& Zimmer-Gembeck, 2016). O presente estudo, pautado na preocupação com a popularização do conhecimento científico, segue também as indicações para a elaboração de cartilhas de orientação aos cuidados em saúde, buscando o conhecimento científico sobre o tema. Ainda, foram escolhidas uma linguagem acessível e informações relevantes, incluindo a participação de leigos e de profissionais da saúde e áreas afins, e mantendo o rigor científico indispensável para garantir a qualidade do produto (Echer, 2005)

As sugestões da Cartilha foram elaboradas com base em materiais já disponíveis sobre saúde mental frente ao estresse e à COVID-19 (CDCP, 2002; Lunn et al., 2020; OPAS, 2015; Park \& Park, 2020; Shonkoff, 2020; van Bavel et al., 2020; WHO, 2020; Williams \& Fletch, 2003). Apoiam-se também em algumas abordagens das Terapias Comportamentais Contextuais (Lucena-Santos, Pinto-Gouveia, \& Oliveira, 2015), as quais se baseiam na premissa de que um comportamento só pode ser entendido a partir da compreensão do contexto. Dentre as abordagens das Terapias Comportamentais Contextuais, foram utilizadas as de mindfulness e autocompaixão (Dorjee, 2016; Lucena-Santos et al., 2015) e, principalmente, o escopo teórico e prático da Terapia Comportamental Dialética (Dialetical Beharior Therapy), desenvolvida especialmente para pessoas com desregulação emocional (Cavalheiro \& Melo, 2016).

Nesse sentido, incluir algumas estratégias de regulação emocional na Cartilha, de forma adaptada, bem como ter o escopo teórico da Terapia Comportamental Dialética como base do material desenvolvido, é coerente com a proposta de coping como autorregulação sob estresse. Cabe ressaltar que a Cartilha é voltada para o público geral em contexto de epidemia, e não especificamente para pessoas com problemas de regulação emocional. Já as abordagens com base na compaixão e autocompaixão proporcionam uma postura sensível e acolhedora ao sofrimento próprio e alheio. Estar consciente da própria experiência, ser gentil consigo mesmo ou com o outro, ao invés de ser crítico, e ter o senso de que todos os seres humanos são passíveis de sofrimento e de falha, pode proporcionar uma forma mais saudável/adaptativa de lidar com

4 situações difíceis e estressantes (Dorjee, 2016; Neff \& Germer, 2019). 


\section{Avaliação do conteúdo da Cartilha por juízes}

Participaram da avaliação da Cartilha oito juízes, sendo seis do sexo feminino (75\%) e dois do sexo masculino (25\%). A média de idade foi de 48,2 anos ( $S D=12,6)$, com amplitude de 28 a 65 anos de idade. Quanto à área de atuação, quatro eram psicólogos, um administrador, um biólogo, um educador físico e um fisioterapeuta. Quanto ao nível máximo de instrução, dois eram graduados em curso superior, um era especialista, quatro mestres e um doutor.

Foi solicitado aos juízes que avaliassem o conteúdo da Cartilha, dividido em seções: introdução, com identificação de sinais de estresse e ansiedade; quadro sobre a NPB de competência; quadro sobre a NPB de relacionamento; e quadro sobre a NPB de autonomia. A avaliação teve foco na clareza e na pertinência do conteúdo das seções. O critério de clareza diz respeito à linguagem empregada e sua compreensibilidade, ao passo que o critério de pertinência avalia a utilidade e adequação do conteúdo. Ainda foi fornecido um espaço para os juízes acrescentarem comentários ou sugestões de modificação. Por fim, a versão final elaborada pelos autores foi tratada por trabalho de designer gráfico, realizado gratuitamente pela empresa de consultoria Wisnet Consulting Ltda. Foi licenciada no Creative Commons, tendo acesso livre.

\section{Resultados e Discussão}

\section{Avaliação dos juízes}

Os juízes ( $N=8$ ) avaliaram, com 100,0\% de concordância, que o conteúdo das seções da Cartilha possuía clareza e pertinência. Sobre as sugestões de modificação, quatro juízes $(50,0 \%)$ sugeriram acréscimo de conteúdo (e.g., sintoma de pânico, estratégia de exercício físico), três (37,7\%) sugeriram pequenas alterações de linguagem (e.g., mudar "crianças sozinhas" para "criança sozinhas em casa"), um juiz (12,5\%) sugeriu mudanças no layout (e.g., formato de checklist, espaço para anotações), e um juiz (12,5\%) indicou que partes do texto estavam repetitivas. As sugestões dos juízes foram acatadas pelos autores e o conteúdo da Cartilha revisado conforme indicado.

\section{A Cartilha}

O material produzido foi denominado "Cartilha para enfrentamento do estresse em tempos de pandemia". Contém uma seção introdutória, que descreve questões gerais sobre o cenário atual em relação a aspectos psicológicos do estresse. Segue-se uma seção com a identificação de reações comuns de estresse segundo a literatura da área (Aldwin, 2009; Lazarus \& Folkman, 1984), bem como a indicação de serviços onde buscar auxílio em casos mais graves. A terceira seção da Cartilha trata das estratégias de enfrentamento referentes a cada uma das três NPB: competência, relacionamento e autonomia. Para cada uma delas, são apresentados três quadros, apontando "Dificuldades", "O que evitar" e "O que posso fazer". O quadro "Dificuldades" apresenta uma relação dos estressores que as pessoas podem estar vivendo, segundo dados da literatura específica sobre reações à própria pandemia e a outras epidemias e desastres (Afifi et al., 2012; Aldwin, 2009; Barros-Dalben et al., 2020; Benight \& Harper, 2002; Brooks et al., 2020; CDCP, 2020; Park \& Park, 2020). O segundo quadro, denominado "O que evitar", contém uma explicação e exemplos das estratégias de enfrentamento com desfechos menos adaptativos em termos de saúde física e mental, normalmente apresentadas pelas pessoas, segundo revisão da área (Skinner et al., 2003). O último 
quadro da terceira seção, "O que posso fazer", contém as estratégias de enfrentamento adaptativas e foi elaborado com base na literatura sobre intervenções psicológicas em contexto de estresse (Dorjee, 2016; Florko, 2020; Hanssen et al., 2015; Linehan, 2018; Lunn et al., 2020; Neff \& Gerner, 2019), indicadas por órgãos e instituições internacionais (CDCP, 2020; OPAS, 2015; Park \& Park, 2020; Shonkoff, 2020; Williams \& Fletch, 2003; WHO, 2020).

Os quadros de estratégias de enfrentamento foram organizados de acordo com cada uma das três NPB, considerando tanto a percepção de ameaça (associada a estratégias de enfrentamento com desfechos mal adaptativos) quanto a percepção de desafio (com desfechos adaptativos no médio e longo prazo):

(i) Competência - O processo adaptativo em jogo envolve a coordenação de ações e contingências, promovendo a percepção do controle de si e da situação, bem como do senso de autoeficácia. A percepção de ameaça à NPB de competência leva a estratégias de enfrentamento relacionadas ao "Desamparo", pois a pessoa entende que suas ações são limitadas e inefetivas, gerando passividade, desânimo, confusão, interferência e exaustão cognitiva, por exemplo. A percepção de ameaça a essa NPB associa-se também à "Fuga" de ambientes não contingentes, com afastamento mental, negação, evitação e pensamento mágico, dentre outros. Por outro lado, as estratégias podem também relacionar-se à percepção de desafio a NPB de Competência, sendo classificadas como estratégias de "Resolução de Problemas", nas quais a pessoa procura ajustar suas ações em busca de efetividade, por meio de planejamento de estratégias, ação instrumental, domínio. Também apresenta estratégias de enfrentamento relacionadas à "Busca de Informações", em que procura contingências adicionais, por meio de leitura, observação do outro, perguntas, consulta a especialistas etc.;

(ii) Relacionamento - O processo adaptativo atuante envolve a coordenação da confiança e dos recursos sociais disponíveis. A percepção de ameaça à NPB de relacionamento associa-se a estratégias de enfrentamento com a função de "Delegação", o que é indicativo de que a pessoa avalia ter limitações para utilizar recursos sociais, apresentando comportamentos como reclamar, autoculpar-se, lamentar-se. Relacionase também ao "Isolamento Social", com o afastamento de contextos sociais não apoiadores, por meio de evitação do outro, dissimulação, "congelamento"/paralisia. A percepção de desafio a essa NPB, por sua vez, associa-se a estratégias de enfrentamento classificadas como indicativas de "Autoconfiança", procurando proteger os recursos sociais disponíveis, com regulação emocional e comportamental, expressão e aproximação emocional. Também se associa à "Busca de Suporte Social", usando os recursos sociais disponíveis, com busca de contato, busca de conforto, ajuda instrumental e referenciamento social;

(iii) Autonomia - O processo adaptativo em ação consiste na coordenação de preferências e opções disponíveis. A percepção de ameaça à NPB de autonomia pode levar a estratégias de enfrentamento relacionadas à "Submissão", que ocorre quando a pessoa desiste de suas preferências, apresentando ruminação, pensamentos intrusivos e perseveração rígida. As estratégias de enfrentamento podem também ter a função de "Oposição", quando os obstáculos são removidos de forma desajustada, com a presença de comportamentos agressivos e desafiadores, como culpar os outros ou projetar no outro, por exemplo. De outro lado, a percepção de desafio a essa NPB se associa a estratégias de enfrentamento relacionadas à "Acomodação", em que há um ajuste flexível das opções, com estratégias de enfrentamento como distração cognitiva, reestruturação cognitiva, minimização e aceitação. Ocorrem também estratégias de enfrentamento relacionadas à "Negociação", quando a pessoa procura encontrar novas opções por meio de barganha, persuasão e estabelecimento de prioridades (Skinner et al., 2003; Skinner \& Wellborn, 1994).

Por fim, "Minhas Anotações" apresenta espaço para o leitor escrever suas opções pessoais sobre como agir. Em outros campos da Cartilha, há espaço para marcações que tornam o material mais interativo. Para motivar a leitura dos quadros, o leitor é desafiado não somente a avaliar como está atualmente respondendo 6 aos estressores, mas a entrar em contato com outras possibilidades de resposta diante da situação. Também 
visando promover a motivação do leitor, o conteúdo foi ilustrado com figuras e quadros coloridos, dispostas em cada uma das dezesseis páginas que compuseram a Cartilha, com uma proposta interativa incluindo check dots e espaço para anotações.

O trabalho final de Weide et al. (2020) está disponível oficialmente nos sites das duas universidades envolvidas no Projeto: (a) PUC-Campinas: <https://www.puc-campinas.edu.br/wp-content/uploads/2020/04/ cartilha-enfrentamento-do-estresse.pdf.pdf z; e (b) PUCRS: <http://www.pucrs.br/wp-content/ uploads/2020/04/Cartilha-para-Enfrentamento-do-Estresse-em-Tempos-de-Pandemia.pdf $>$. Esclarece-se, ainda, que a Cartilha foi disponibilizada antes do artigo para atender com urgência a uma necessidade de utilidade pública.

\section{Considerações Finais}

Com base em evidências científicas da área da Psicologia, foi gerado como produto um material de apoio, no formato de uma Cartilha fundamentada em conceitos psicológicos bem estabelecidos e que poderá auxiliar no enfrentamento do estresse, orientando sobre como lidar com os problemas emocionais e comportamentais relacionados à pandemia de COVID-19. Tem como diferencial ter uma estrutura organizada, coerente do ponto de vista teórico e metodológico, apta a transferir conhecimento para a prática profissional, consistindo, ainda, em um material útil para a população em geral. Assim, foram mostradas algumas das possibilidades de aplicação da Psicologia no campo da Saúde.

Essa produção serviu, também, como base para a instalação de uma força-tarefa emergencial, denominada PsiCOVIDa, fundada pelos autores com o intuito de gerar outros produtos de comunicação e orientação para uso público durante o período crítico da pandemia da COVID-19. Dentro dessa proposta de ações interinstitucionais, foi elaborada esta Cartilha em parceria de dois programas de pós-graduação em Psicologia. Espera-se que esta produção possa servir de base para futuros estudos e como material para uso em serviço de saúde, além de ser utilizada pelo público em geral e como apoio às ações dos profissionais da saúde.

\section{Agradecimentos}

Ao Conselho Nacional de Desenvolvimento Científico e Tecnológico (CNPq); Coordenação de Aperfeiçoamento de Pessoal de Nível Superior (Capes) e Wisnet Consulting Ltda. pelo trabalho gráfico realizada pelo designer G. F. COSTA.

\section{Colaboradores}

Todos os autores participaram da concepção e desenho; análise e interpretação dos dados e discussão dos resultados, e revisão e aprovação da versão final do artigo.

\section{Referências}

Afifi, W. A., Felix, E. D., \& Afifi, T. D. (2012). The impact of uncertainty and communal coping on mental health following natural disasters. Anxiety, Stress \& Coping, 25(3), 329-347. http://dx.doi.org/10.1080/10615806.2011.603048

Aldwin, C. M. (2009). Stress, coping and development: an integrative perspective (2nd ed.). New York: The Guilford Press.

Barros-Delben, P., Cruz, R. M., Trevisan, K. R. R., Gai, M. J. P., Carvalho, R. V. C., Carl, P. A. C., ... Malloy-Diniz, L. F. (2020). Saúde mental em situação de emergência: COVID-19 [Ahead of print]. Revista Debates in Psychiatry, 10. Recuperado de https://d494f8133c95463a898cea1519530871.filesusr.com/ugd/c37608_e2757d5503104506b30e50caa6fa6aa7.pdf 
Benight, C. C., \& Harper, M. L. (2002). Coping self-efficacy perceptions as a mediator between acute stress response and long-term distress following natural disasters. Journal of Traumatic Stress, 15(3), 177-186. http://dx.doi. org/10.1023/A:1015295025950

Brooks, S. K., Webster, R. W., Smith, L. E., Woodland, L., Wessely, S., Greenberg, N., \& Rubin, G. J. (2020). The psychological impact of quarantine and how to reduce it: rapid review of the evidence. The Lancet, 395(10227), 912-920. http:// dx.doi.org/10.1016/S0140-6736(20)30460-8

Cavalheiro, C. V., \& Melo, W. (2016). Relação terapêutica com pacientes. Psicologia em Revista, 22(3), 579-595. http:// dx.doi.org/10.5752/P.1678-9523.2016V22N3P579

Center for Disease Control and Prevention. (2020). Stress and coping. Atlanta: Author. Retrieved from https://www.cdc. gov/coronavirus/2019-ncov/daily-life-coping/managing-stress-anxi ety.html?CDC_AA_refVal=https\%3A\%2F\%2Fwww. cdc.gov\%2Fcoronavirus\%2F2019-ncov\%2Fprepare\%2Fmanaging-stress-anxiety.html

Compas, B. E., Connor-Smith, J. K., Saltzman, H., Thomsen, A. H., \& Wadsworth, M. E. (2001). Coping with stress during childhood and adolescence: problems, progress, and potential in theory and research. Psychological Bulletin, 127(1), 87-127. http://dx.doi.org/10.1037/0033-2909.127.1.87

Correia, S., Luck, S., \& Verner, E. (2020). Pandemics depress the economy, public health interventions do not: evidence from the $1918 \mathrm{Flu}$. Social Science Research Network. http://dx.doi.org/10.2139/ssrn.3561560

Dorjee, D. (2016). Defining contemplative science: the metacognitive self-regulatory capacity of the mind, context of meditation practice and modes of existential awareness. Frontiers in Psychology, 17(1788). http://dx.doi.org/10.3389/ fpsyg.2016.01788

Echer, I. C. (2005). Elaboração de manuais de orientação para o cuidado em saúde. Revista Latino-Americana de Enfermagem, 13(5),754-757. http://dx.doi.org/10.1590/\$0104-11692005000500022

Enumo, S. R. F., Linhares, M. B. M., Machado, W. A., \& Silva, A. M. B. (2017). Impacto do estilo de vida sobre as condições de saúde do adulto. In M. A. Santos, D. Bartholomeu \& J. M. Montiel (Orgs.), Relações interpessoais no ciclo vital: conceitos e contexto (pp.313-334). São Paulo: Vetor.

Florko, L. (2020, March 23). Meeting your needs while working from home: how to ensure you stay psychologically healthy [Web log post]. New York: Psychology Today. Retrieved from https://www.psychologytoday.com/ca/blog/ people-planet-profits/202003/meeting-your-needs-while-working-home

Folkman, S. (2011). The Oxford handbook of stress, health, and coping. New York: Oxford University Press.

Hanssen, M. M., Vancleef, L. M. G., Vlaeyen, J. W. S., Hayes, A. F., Schouten, E. G. W., \& Peters, M. L. (2015). Optimism, motivational coping and well-being: evidence supporting the importance of flexible goal adjustment. Journal of Happiness Studies, 16, 1525-1537. http://dx.doi.org/10.1007/s10902-014-9572-x

Inter-Agency Standing Committee. (2020). Guia preliminar: como lidar com os aspectos psicossociais e de saúde mental referentes ao surto de COVID-19 - Versão 1.5. Genebra: Autor. Recuperado de https://interagencystandingcommittee. org/system/files/2020-03/IASC \%20Interim\%20Briefing\%20Note\%20on \%20COVID-19\%20Outbreak\%20 Readiness \%20and\%20Response\%200perations\%20-\%20MHPSS\%20\%28Portugue se\%29.pdf

Keyes, C. L. M. (2007). Promoting and protecting mental health as flourishing: a complementary strategy for improving national mental health. American Psychologist, 62(2), 95-108. http://dx.doi.org/10.1037/0003-066X.62.2.95

Lazarus, R. S., \& Folkman, S. (1984). Stress, appraisal and coping. New York: Springer Publishing Company.

Liang, T. (2020). Zhejiang University School of Medicine. Handbook of COVID-19: prevention and treatment. Paris: Unesco. Retrieved from http://www.zju.edu.cn/english/2020/0323/c19573a1987520/page.htm

Linehan, M. M. (2018). Treinamento de habilidades em DBT: manual de terapia comportamental dialética para o paciente (2a ed.). Porto Alegre: Artmed.

Lucena-Santos, P., Pinto-Gouveia, J., \& Oliveira, M. S. (2015). Terapias comportamentais de terceira geração: guia para profissionais. Porto Alegre: Synopsis Editora.

Lunn, P., Belton, C., Lavin, C., McGowan, F., Timmons, S., \& Robertson, D. (2020). Using behavioural science to help fight the coronavirus. Dublin: Economic and Social Research Institute. Retrieved from https://www.esri.ie/system/files/ publications/WP656.pdf

Melnik, T., Souza, W. F., \& Carvalho, M. R. (2014). A importância da prática da Psicologia baseada em evidências: aspectos conceituais, níveis de evidência, mitos e resistências. Revista Costarricense de Psicología, 33(2), 79-92. Recuperado de http://rcps-cr.org/wp-content/themes/rcps/descargas/2014/2/0-RCP-Vol.33-No2.pdf 
Miller, G. E., Chen, E., \& Parker, K. J. (2011). Psychological stress in childhood and susceptibility to the chronic diseases of aging: moving towards a model of behavioral and biological mechanisms. Psychological Bulletin, 137(6), 959-997. http://dx.doi.org/10.1037/a0024768

Neff, K., \& Germer, C. (2019). Manual de mindfulness e autocompaixão: um guia para construir forças internas e prosperar na arte de ser seu melhor amigo. Porto Alegre: Artmed.

Organização Panamericana de Saúde. (2015). Primeiros cuidados psicológicos: guia para trabalhadores de campo (M. Gagliato, Trad.). Brasília: Autor. Recuperado de https://www.paho.org/bra/index.php?option=com docman\&view=download\&category_slug=prevencao-e-cont-doencas-e-desenv-sustentavel-071\&alias=1517-primeiroscuidados-psicologicos-um-guia-para-trabalhadores-campo-7\&ltemid=965

Park, S. C., \& Park, Y. C. (2020). Mental health care measures in response to the 2019 novel coronavirus outbreak in Korea. Psychiatry Investigation, 17(2), 85-86. http://dx.doi.org/10.30773/pi.2020.0058

Qiu, J., Shen, B., Zhao, M., Wang, Z., Xie, B., \& Xu, Y. (2020). A nationwide survey of psychological distress among Chinese people in the COVID-19 epidemic: implications and policy recommendations. General Psychiatry, 33, e100213. http://dx.doi.org/10.1136/gpsych-2020-100213

Ramos, F. P., Enumo, S. R. F., \& Paula, K. M. P. (2015). Teoria Motivacional do Coping: uma proposta desenvolvimentista de análise do enfrentamento do estresse. Estudos de Psicologia (Campinas), 32(2), 269-280. http://dx.doi.org/10. 1590/0103-166X2015000200011

Ryan, R. M., \& Deci, E. L. (2017). Self-determination theory: basic psychological needs in motivation, development and wellness. New York: The Guilford Press.

Seligman, M. E. P., \& Csikszentmihalyi, M. (2000). Positive Psychology: an introduction. American Psychologist, 55(1), 5-14. http://dx.doi.org/10.1037/0003-066X.55.1.5

Shonkoff, J. (2020). Stress, resilience, and the role of science: responding to the coronavirus pandemic. Cambridge: Center on Developing Child. Retrieved from https://developingchild.harvard.edu/stress-resilience-and-the-role-ofscience-responding-to-the-coronavirus-pandemic/

Shonkoff, J. P., Garner, A. S., Committee on Psychosocial Aspects of, Child, Family, Health, Committee on Early Childhood, Adoption, Dependent, Care, Behavioral Pediatrics. (2012). The lifelong effects of early childhood adversity and toxic stress. American Academy of Pediatrics, 129(1), e232-e246. http://dx.doi.org/10.1542/peds.2011-2663

Skinner, E. A, Edge, K., Altman, J., \& Sherwood, H. (2003). Searching for the structure of coping: a review and critique of category systems for classifying ways of coping. Psychological Bulletin, 129(2), 216-269. http://dx.doi.org/10.1037/00332909.129.2.216

Skinner, E. A., \& Wellborn, J. G. (1994). Coping during childhood and adolescence: a motivational perspective. In D. L. Featherman, R. M. Lerner, \& M. Perlmutter (Eds.). Life-Span development and behavior (pp.91-133). Hillsdale: Lawrence Erlbaum Associates.

Skinner, E. A., \& Zimmer-Gembeck, M. J. (2016). The development of coping: stress, neurophysiology, social relationships, and resilience during childhood and adolescence. New York: Springer.

Slavich, G. M., \& Cole, S. W. (2013). The emerging field of Human Social Genomics. Clinical Psychological Science, 1(3) 331-34. http://dx.doi.org/10.1177/2167702613478594

van Bavel, J. J., Boggio, P. S., Capraro, V., Cichocka, A., Cikara, M., Crockett, M. J., ... Willer, R. (2020). Using social and behavioural science to support COVID-19 pandemic response. Nature Human Behavior. http://dx.doi.org/10.1038/ s41562-020-0884-z

Weide, J. N., Vicentini, E. C. C., Araujo, M. F., Machado,W. L., \& Enumo, S. R. F. (2020). Cartilha para enfrentamento do estresse em tempos de pandemia. Campinas: Puc-Campinas. Recuperado de https://www.puc-campinas.edu.br/ wp-content/uploads/2020/04/cartilha-enfrentamento-do-estresse.pdf.pdf

Williams, R. T., \& Fetsch, R. J. (2003). Farm and ranch family stress and depression: a checklist and guide for making referrals. Retrieved from https://agrilifeextension.tamu.edu/wp-content/uploads/2016/01/Farm-Ranch-Family-StressDepression-Checklist-for-Making-Referrals.pdf

World Health Organization. (2004). Promoting mental health: concepts, emerging evidence, practice. Geneva: Author. Retrieved from https://www.who.int/mental_health/evidence/en/promoting_mhh.pdf

World Health Organization. (2020). Mental health and psychosocial considerations during the COVID-19 outbreak. Geneva: Author. Retrieved from https://www.who.int/docs/default-source/coronaviruse/mental-healthconsiderations.pdf 
Zimmer-Gembeck, M. J., \& Skinner, E. A. (2011). The development of coping across childhood and adolescence: an integrative review and critique of research. International Journal of Behavioral Development, 35, 1. http://dx.doi. org/10.1177/0165025410384923

Zimmer-Gembeck, M. J., \& Skinner, E. A. (2016). The development of coping and regulation: implications for psychopathology and resilience. In D. Cicchetti (Ed.), Developmental psychopathology (3rd, pp.485-544). New York: Wiley. Retrieved from http://au.wiley.com/WileyCDA/WileyTitle/productCd-1118121791.html

Zimmer-Gembeck, M. J., Skinner, E. A., Modecki, K. L., Webb, H. J., Gardner, A. L., Hawes, T., \& Rapee, R. M. (2018). The self-perception of flexible coping with stress: a new measure and relations with emotional adjustment. Cogent Psychology, 5, 1537908. http://dx.doi.org/10.1080/23311908.2018.1537908

Recebido: abril 7, 2020

Aprovado: abril 17, 2020 